\title{
XI INTERNATIONAL CONGRESS OF DERMATOLOGY
}

\author{
STOCKHOLM, 1957
}

The XI International Congress of Dermatology was held in Stockholm under the presidency of Professor Sven Hellerström, from July 31 to August 6, 1957 , and was attended by some hundreds of dermatologists and venereologists from all parts of the world. Symposia were conducted on four subjects of direct interest to venereologists:

(1) Specific syphilis serology;

(2) Epidemiology of the treponematoses;

(3) Long term results in the treatment of syphilis;

(4) Non-gonococcal urethritis;

Unfortunately the discussions on serology and epidemiology were held in different lecture rooms at the same time, so that it was not possible to attend both.

(1) Specific Syphilis Serology.-Among the subjects discussed was the complement-fixation test for syphilis using as antigen a protein fraction derived from $T$. pallidum (Reiter's strain), as originally evolved by D'Alessandro and Puccinelli. Dr. J. H. de Bruijn (Utrecht) described a comparison between this test and the treponemal immobilization test. It appeared that the complement-fixation test with protein antigen was both sensitive and specific. Drs. Bokkenheuser and Kooij (South Africa) gave results with standard serological tests (STS) for syphilis and treponemal immobilization tests (TPI) on the sera of patients suffering from leprosy. False positive standard STS were found exclusively in cases of leprosy of the lepromatous type and not in those of the tuberculoid type. Dr. Catterall (London) described findings in 41 cases of biological false positive reaction to standard tests for syphilis. The results were substantially the same as those described by Moore and Lutz. The British Cooperative Clinical Group presented a study, prepared by Dr. A. E. Wilkinson, in which information concerning pregnant women whose routine serological tests for syphilis were positive was collected by means of a questionnaire completed by the directors of 47 clinics, and was submitted to analysis. In general, the results of the TPI test were in good agreement with the clinical assessment, but divergence was greater in TPI-negative cases. Although the tests were performed on a selected group, it seemed probable that an appreciable proportion of positive STS found on routine antenatal testing were nonspecific, and it was expected that with the diminishing incidence of syphilis, this proportion would increase in the future. It was emphasized that the ultimate responsibility for the assessment of a positive STS as being non-specific rests on the physician and not on the serologist. The diagnosis is reached by exclusion after thorough investigation of the patient and her family. The TPI test can play an important part in this, but it has limitations which must be clearly recognized; it should not be used as a short cut to exclude the necessity for careful investigation. Dr. Chacko (Madras) provided information concerning skin tests for syphilis using an antigen containing $T$. pallidum of the Nichol's strain. Dr. Coster (Utrecht) compared standard tests and the TPI test with a treponemal agglutination test using the Nichol's strain of $T$. pallidum. The test was sensitive and specific, but there was a liability for the treponemes to become sensitized-probably at the time of extraction from the rabbits' testes. There was no certain way of avoiding this difficulty. Dr. Daguet (Paris) found the TPI test so specific and sensitive that he believed there was a case for considering it the standard sero-diagnostic reaction for syphilis. He found there was close correlation between the TPI test and the Treponemal Immune Adherence Test. Complement-fixation tests with antigen prepared from Reiter's treponemes were more specific than complement-fixation tests with cardiolipin antigen.

(2) Epidemiology of the Treponematoses.-The discussion of this subject covered a wide field. Dr. Simons (Amsterdam) referred to the relationship between syphilis and the non-venereal treponematoses. Two of the latter were confined to the tropics and were unable to spread in colder countries. Their incidence was quite certainly associated with rain. Their precise relationship to syphilis remained doubtful. He believed that, in those areas where the incidence of these diseases was higher than 20 per 
cent., everyone should be regarded as a contact and should be treated. The World Health Organization deserved great credit for its epidemiological and clinical work in this field, but extended effort was essential because the deleterious effect of bad economic conditions lasted longer that the effect of antibiotics. Dr. DeLamater (Philadelphia) described the life cycles of treponemes in relation to the pathogenesis of the diseases which they caused. Dr. Danbolt $(O s l o)$ reviewed the results of re-study of the Boeck-Bruusgaard cases of untreated syphilis. Dr. McElligott (London) described the decline in the incidence of early syphilis in the United Kingdom. In 1955, new cases were less than 5 per cent. of those in 1946. Late and latent syphilis had fallen consistently since 1952 . Cardiovascular syphilis began to fall after 1955. Dr. Rajam (Madras) estimated that 4 to 6 per cent. of the population of Madras had positive serological tests for syphilis. The cases included all forms of syphilis and yaws, and some of non-venereal syphilis among slum-dwellers. Dr. Takenouchi (Chiba, Japan) related the incidence of syphilis in Japan to differing social and economic conditions. The latter were at their lowest ebb in 1946 and 1947, and the incidence of syphilis was then highest, that of early syphilis being more than 50 per cent. of all cases of syphilis. Syphilis began to decline soon after 1947, even before antibiotics came into general use. This was evidence that social and economic stability was a decisive factor in the control of venereal disease.

(3) Long-Term Results in the Treatment of Syphilis.-Dr. Clarence Smith and Miss Price, of the United States Public Health Service, gave an up-todate review of two important studies which are proceeding in the United States. The first, the socalled "Blue Star" study, begun in 1945, comprised an intensive follow-up of 956 patients treated with penicillin alone for dark-field-positive primary or secondary syphilis, at some time during the period 1946 to 1954 . Additional treatment was required in 138 cases, but it was thought that re-infection had occurred in two-thirds of these. Only one patient progressed to late syphilis and in only four cases was re-treatment necessary because of changes in the cerebrospinal fluid. After a single course of penicillin the cumulative re-treatment rate for primary syphilis was 13 per cent., and for secondary syphilis 16 per cent. 735 patients were observed for 4 years or longer, and for these the average duration of observation was 6.6 years. 98 per cent. of patients initially treated for primary syphilis and 96 per cent. initially treated for secondary syphilis were serologically negative at the last examination. More than
90 per cent. of the patients were treated with outmoded types of penicillin or with less than the minimum recommended dosage. Up-to-date information indicated that a single injection of 2,500,000 units benzathine penicillin $G$. was equally as effective as a single course of injections of procaine penicillin and aluminium monostearate, totalling 4,800,000 units, the latter preparation being the generally accepted remedy of choice. The second investigation, begun in 1943, was designed to study the effectiveness of penicillin in the treatment of neurosyphilis. Posttreatment observation and tests of 765 patients suffering from symptomatic neurosyphilis indicated that the cumulative probability of clinical progression was 0.55 per cent. for the first year, 2 per cent. for the fourth year, and 3.31 per cent. for the seventh year, after treatment with penicillin. In contrast, among 467 patients with asymptomatic neurosyphilis treated with metal chemotherapy, there were 44 instances of clinical progression. Experience with 1,086 cases of general paralysis, observed for 3 years or more in 79 per cent. of the cases, indicated that early diagnosis and prompt treatment with penicillin would practically always prevent progression and death from the disease. Drs. Chargin, Vandon, Sobel, and Rosenthal (New York), compared results obtained in fifty cases of early syphilis treated with penicillin alone and observed for 5 to 11 years, with 72 cases treated with arsenic and bismuth alone and observed for similar periods. The results confirmed findings of others that treatment with penicillin is superior in all respects to that with arsenicals and heavy metals. Drs. McElligott and Willcox (London) reviewed the results obtained in 864 cases of early syphilis, of which 561 received penicillin plus arsenic and bismuth, 183 penicillin and bismuth, and 120 penicillin alone. The results were excellent in all groups and seemed, if anything, rather better with penicillin alone. This was possibly due to the fact that higher doses of penicillin were given for longer periods when no adjuvant therapy was given, and also to the fact that penicillin alone had been used more recently, when the incidence of the disease had fallen and the chances of re-infection were less. Penicillin alone was confidently recommended as the treatment of choice for early syphilis. Drs. Joulia, Texier, Léonard, and Tasei (Bordeaux), described excellent results in the treatment of relatively few cases of early syphilis. Their results confirmed the efficacy of treatment with penicillin alone, even in relatively small doses. Prof. Degos and Dr. Ebard (Paris) described satisfactory results from the treatment of early syphilis with penicillin, but believed that sufficient time had not yet elapsed for full assessment of this drug. Adjuvant treatment 
with bismuth provided an additional guarantee, especially in cases for which subsequent surveillance was difficult or impossible, and in those, e.g. prostitutes, in which there was a serious risk of spread of disease if cure was not complete. Dr. Beerman (Philadelphia), described results of the treatment of cardiovascular syphilis and neurosyphilis with penicillin and gave details of recommended dosage and methods of adminstration. Dr. Grin (Sarajevo), discussed the epidemiological factors concerned in the elimination of non-venereal syphilis in communities showing a high incidence of this disease. He reported that the campaign sponsored by the World Health Organization had resulted in the virtual elimination of this disease in the republic of Bosnia during the past 5 years.

(4) Non-Gonococcal Urethritis.-The chairman, Mr. A. H. Harkness (London), referred to this as the major problem among the venereal diseases. The aetiology of the large majority of these cases was unknown, in spite of much research. The only advance in recent years had been the recognition that more cases were due to the protozoon, Trichomonas vaginalis, than had formerly been supposed. Treatment could only be empirical until the causative organism was discovered. Drs. Nicol and Whittington (London) compared the results of investigation of urethral discharge in the male for the presence of Trichomonas vaginalis by smear and culture at two clinics, showing that by prolonged search of smears and by careful cultural technique it was possible to find the organism in appreciably more cases than with less exacting standards. Dr. Siboulet (Paris) had found 73 cases of trichomonal urethritis among 1,097 patients with urethritis. Smears stained with Giemsa, and cultural methods were almost equally effective in making this diagnosis. Viral inclusions were found in 113 of 2,847 cases of uncomplicated urethritis, and in 31 of 886 cases in which the urethritis was complicated by arthritis.

Drs. Towpik and Stepniak (Warsaw) had made a detailed study of 500 patients selected from 2,500 suffering from non-gonococcal urethritis. Urological complications, including such lesions as Littritis, prostatitis, epididymitis, urethral stricture, and vesiculitis, were found in 35 per cent. Localized focal infections of this kind were the commonest causes of the chronic resistant course which the disease sometimes took.

Dr. Oates (London) reviewed the methods which may be applied for the diagnosis of chronic prostatitis, and produced evidence that the examination by dark-field microscopy of five specimens of prostatic fluid taken from the same material on the same occasion was a far more accurate method of diagnosis than the conventional single wet film or stained smear. He had applied this test to a group of men without history, symptoms, or signs of genital infection, and 15 per cent. of them were found to have chronic prostatitis. Of another group with chronic or relapsing non-specific urethritis, 95 per cent. had evidence of chronic prostatitis. A third group was composed of men suffering from their first attack of non-specific urethritis, and 58 per cent. had evidence of prostatitis. He gave evidence to suggest that the prostate was the main focus of the infection in chronic and relapsing cases. Mr. King (London) summarized the findings in the cases of 100 patients who had experienced 175 attacks of arthritis associated with venereal urethritis. So-called non-specific genital infection sometimes resulted in complications, including the triple syndrome of urethritis, arthritis, and conjunctivitis, often called Reiter's syndrome. It might present with complications and without urethritis, and the diagnosis might then be missed. Arthritis might involve any joints, but was most common in the lower limbs. Residual effects included ankylosing spondylitis, deformities of the feet, painful feet, and sacro-iliac disease. The infection and its complications were very prone to recurrent relapses, with variable intervals, sometimes of years, between the episodes. The frequent association of sacro-iliac disease and relapsing iritis was noted.

Dr. Csonka (London) gave some details of 185 cases of arthritis associated with urethritis, studied over a period of 15 years. The incidence of this condition in relation to the total number of urethral infections seen in the same clinic was 0.6 per cent. Drs. Reynolds and Csonka (London) described the radiological changes seen in this condition. Dr. Willcox (London) reported results in the treatment of uncomplicated non-gonococcal urethritis with various antibiotics. Novobiocin (Streptonivicin, Albamycin, Carthomycin, Cathocin, Cardelmycin) proved ineffective, as did also Aminitrazole (2-acetylamino-5-nitrothiazole). Good results were obtained with Spiramycin (Rovamycin).

The Congress was notable for excellent organization, which was clearly the result of careful and detailed planning, and for the great kindness and courtesy of our Swedish hosts. Stockholm is a pleasant and a beautiful city at any time, but on this occasion the attention to detail and the immense consideration which had been given to the comfort and entertainment of the very large number of those who attended the Congress, together with wives and husbands, made it certain that this would be a week that would live long in the memory of those who participated.

A. J. KING 